\title{
User Fairness Scheme with Proportional Fair Scheduling in Multi-user MIMO Limited Feedback System
}

\author{
Hongyu Wang, Weixiao Meng, Trungtan Nguyen \\ School of Electronics and Information Engineering, Harbin Institute of Technology, Harbin, China \\ Email: wanghongyuhit@sina.com,wxmeng@hit.edu.cn,trungtan68@gmail.com
}

Received June, 2013

\begin{abstract}
In Multi-user MIMO (MU-MIMO) downlink system, suitable user selection schemes can improve spatial diversity gain. In most of previous studies, it is always assumed that the base station (BS) knows full channel state information (CSI) of each user, which does not consider the reality. However, there are only limited feedback bits in real system. Besides, user fairness is often ignored in most of current user selection schemes. To discuss the user fairness and limited feedback, in this paper, the user selection scheme with limited feedback bits is proposed. The BS utilizes codebook precoding transmitting strategy with LTE codebook. Furthermore, this paper analyzes the influence of the number of feedback bits and the number of users on user fairness and system sum capacity. Simulation results show that in order to achieve better user fairness, we can use fewer bits for feedback CSI when the number of user is small, and more feedback bits when the number of users is large.
\end{abstract}

Keywords: MU-MIMO; User Scheduling; User Fairness; PFS; Limited Feedback

\section{Introduction}

In Multi-user MIMO downlink system, the BS can independently transmit data streams to different user utilizing the same time-frequency resources. It is called space division multiple access (SDMA), which can bring more throughput due to multi-user division gain. Multi-user MIMO has been included in 3GPPLTE [1] and LTEAdvance standard. In downlink transmit strategy, the dirty paper coding (DPC) has been proved to achieve the optimal capacity in [2]. But in real system, it is difficult to realize due to its high complexity. So some suboptimal schemes are studied, in which the Zero-forcing beamforming (ZFBF) is a classic scheme. It is shown in [3] that in the condition of large number of users, the capacity of ZFBF approaches to DPC.

Full CSI of all the users is required in both the DPC and the ZFBF. Otherwise, the system throughput will reduce severely [4]. However, full CSI requires larger bandwidth to feedback which is unrealistic, since the number of feedback bits is always limited in real-world systems. Currently, most studies focus on improving the sum rate with limited feedback bits [5,6].

In Multi-user MIMO systems, the system sum rate will be severely affected by multi-user interference since the BS transmits to multiple users simultaneously. Accordingly, it is necessary to pick up the users with good spatial orthogonality to transmit. Furthermore, since the number of antenna in the BS is fixed, when the number of user is larger than the number of antennas, user scheduling is needed. User selection can achieve spatial diversity gain, and further increase sum rate in [7]. [8] shows that semi-orthogonal user selection (SUS) can reduce multi-user interference effectively by scheduling multiple users that have small space correlation. However, SUS assumes that the BS knows the CSI completely, which is unavailable in limited feedback systems. Most of multiuser scheduling schemes use the fixed beamforming codebook. The user calculates the signal-to-interferenceplus-noise ratio (SINR) with each codeword and feedback the codeword index which achieves maximum SINR along with the SINR value. Then the BS selects the user with the highest SINR on every codeword and uses the codebook as pre-coding matrix for downlink transmitting. In most user scheduling algorithms, user fairness is not considered, but in practice it is often important to take care of the bad channel users. The PFS provides fairness among users. In $[9,10]$, the user average capacity is considered to avoid the bad channel users not being served for a long time.

In this paper, we consider LTE codebook as the limited feedback codebook. In LTE codebook, the codeword in the same sub-codebook are orthogonal, while the codeword in different sub-codebook are not orthogonal. So the BS must select the codeword in the same sub-codebook as the beamforming weight vector. User 
feedback the codeword index, which achieves the maximum SINR and the corresponding SINR value, the BS schedules users with the max system sum capacity among the sub-codebooks. Using LTE codebook and PFS, we consider the user fairness with different number of feedback bits on the condition of different number of users.

\section{System Model}

In this paper, we consider multi-user MIMO downlink system. Figure 1 shows the system channel model. The BS configures $N$ antennas and each user has single antenna. The BS can communicate with $K(K \leq N)$ users simultaneously. The number of user is $M(K \leq M)$. Denote the channel vector of user $k$ by $\boldsymbol{h}_{k} \in C^{1 \times N}$, where we assume the entries of $\boldsymbol{h}_{k}$ are independent but non-identically distributed complex Gaussian random variables with zero mean. Different variance represents different channel condition. The BS selects a sub-codebook as beamforming matrix $W\left(\in C^{N \times N}\right)$ and form $N$ orthogonal beams. Each beam $\boldsymbol{w}$ is the column vector of $W$. Then the BS communicates to the selected $K$ users simultaneously in one time slot. The receive signal of user $k$ can be expressed as

$$
y_{k}=\sum_{i=1}^{N} P_{k} \boldsymbol{h}_{k} \boldsymbol{w}_{i} s_{i}+n_{k},
$$

where the $s \in C^{N \times 1}$ is the normalized transmitted symbol from the BS which satisfies $E\left\{s s^{*}\right\}=1, n_{k}$ is additive white Gaussian noise (AWGN) vector, and $P_{k}$ is transmit power of the $k^{\text {th }}$ user. The BS has an average power constraint, which is $\sum_{k=1}^{N} P_{k}=P$. In this paper, the channel is time varying Rayleigh fading channel. Assume the user knows its own channel $\boldsymbol{h}_{k}$ perfectly. LTE codebook set is $\left\{\boldsymbol{E}^{(0)}, \cdots, \boldsymbol{E}^{(G-1)}\right\}$, where

$$
\boldsymbol{E}^{(g)}=\left[\begin{array}{lll}
\boldsymbol{e}_{0}^{(g)} & \cdots & \boldsymbol{e}_{N-1}^{(g)}
\end{array}\right] \in C^{N \times N}
$$

is the sub-codebook, and $\boldsymbol{e}_{m}^{(g)} \in C^{N \times 1}$ is the codeword. Then the $k^{\text {th }}$ user computed the SINR over the $m^{\text {th }}$ codeword in the $g^{\text {th }}$ sub-codebook as:

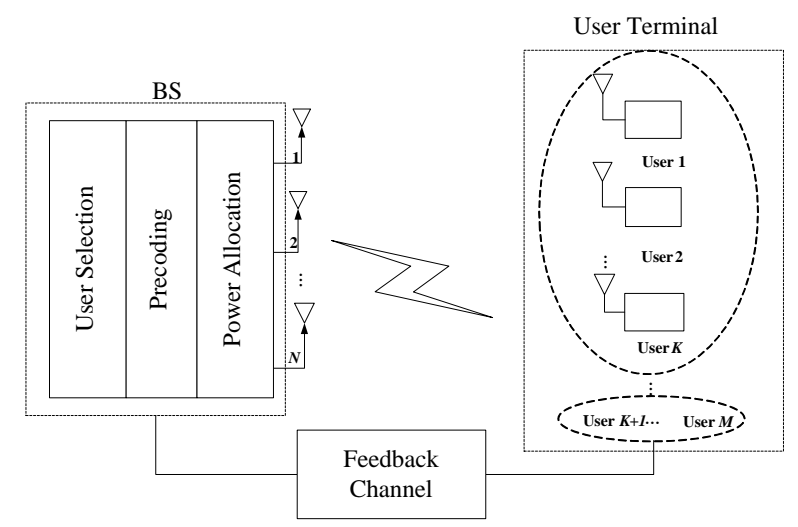

Figure 1. System Channel Model.

$$
\operatorname{SINR}_{k, g, m}=\frac{\left|\boldsymbol{h}_{k} \boldsymbol{e}_{m}^{(g)}\right|^{2}}{1+\sum_{i=1, i \neq m}^{N}\left|\boldsymbol{h}_{k} \boldsymbol{e}_{i}^{(g)}\right|^{2}},
$$

where $k=1,2, \cdots, K, g=1,2, \cdots, G, \quad m=1,2, \cdots, N . G$ is the number of sub-codebook, which decides by the number of feedback bit. Then the terminal user feeds back the maximum $\operatorname{SINR}_{k, g, m}$ and the corresponding codeword index $(g, m)$. The BS selects the maximum SINR user for each codeword. For each sub-codebook $g$, the BS computes the sum rate $R_{g}$ as:

$$
R_{g}=\sum_{m=1}^{N} \log \left(1+\frac{P}{N} \arg \max _{k} \operatorname{SINR}_{k, g, m}\right)
$$

where $P$ is the total transmitting power. In this paper, we just consider equal power allocation. $R_{g}$ is the capacity of the $g^{\text {th }}$ sub-codebook. For $g=1,2, \cdots, G$, the BS selects the maximum $R_{g}$ and the index $g^{*}$.

$$
g^{*}=\arg \max _{g} R_{g}
$$

During the downlink transmission, the BS uses the $\boldsymbol{E}^{\left(g^{*}\right)}$ as the beamforming matrix. So we can get $\boldsymbol{W}=\boldsymbol{E}^{\left(g^{*}\right)}$.

\section{PFS Scheme}

Designed to maximize the sum rate, user scheduling schemes often ignore user fairness, thus making the user with the bad channel seldom served. Therefore, the quality of service (QoS) of these users will not be guaranteed in a QoS sensitive system. The PFS scheme proposed by Jalali and Padovani can solve this problem effectively. The PFS considers the user's current average throughput in a period of time. Using the radio of instantaneous channel quality and average throughput determines whether the user can be scheduled, so it can take account for the tradeoff between throughput and fairness. The criterion of user selection is showed as:

$$
k^{*}=\arg \max _{k=1,2, \cdots, K} \frac{1}{\mu_{k}(t)} R_{k}(t),
$$

where $R_{k}(t)$ is the instantaneous rate of the $k^{\text {th }}$ user, $\mu_{k}(t)$ is the average throughput of the $k^{\text {th }}$ user in past $t_{c}$ time slot which is update by formula (6) every time slot.

$$
\mu_{k}(t+1)=\left\{\begin{array}{cc}
\left(1-\frac{1}{t_{c}}\right) \mu_{k}(t)+\frac{1}{t_{c}} R_{k}(t) & k=k^{*} \\
\left(1-\frac{1}{t_{c}}\right) \mu_{k}(t) & k \neq k^{*}
\end{array}\right.
$$

The traditional PFS before only select one user in each time slot. But in this paper, the BS needs to communicate with more than one user at the same time, so we modify the traditional PFS to adapt to the multi-user MIMO 
case.

In our system, the BS transmits to more than one user simultaneously. But for each codeword, only one user can be selected. So in the $t^{\text {th }}$ time slot, the BS schedules user for the $m^{\text {th }}$ codeword in the $g^{\text {th }}$ sub-codebook using the traditional PFS scheme which can be described as:

$$
k_{g, m}{ }^{*}=\arg \max _{k=1,2, \cdots, K} \frac{1}{\mu_{k}(t)} R_{k, g, m}(t)
$$

where $R_{k, g, m}(t)$ is the instantaneous date rate of the $k^{\text {th }}$ user on the $(g, m)$ codeword, and $\mu_{k}(t)$ is the average data rate of the $k^{\text {th }}$ user in the past $t_{c}$ time slots which is updated as:

$$
\mu_{k}(t+1)=\left\{\begin{array}{cc}
\left(1-\frac{1}{t_{c}}\right) \mu_{k}(t)+\frac{1}{t_{c}} R_{k}(t) & k \text { is scheduled } \\
\left(1-\frac{1}{t_{c}}\right) \mu_{k}(t) & \text { otherwise }
\end{array}\right.
$$

where $R_{k}(t)$ is the instantaneous date rate of user $k$ in $t^{\text {th }}$ time slot.

\section{User Scheduling Process}

In this paper, we choose LTE codebook as local codebook, [1] shows the generation methods of LTE codebook. The number of sub-codebook is decided by the number of feedback bit. And the antenna number in BS determines the number of codeword in a sub-codebook.

The $k^{\text {th }}$ user achieves its channel state information from the downlink pilot channel, and computes the SINR value $\operatorname{SINR}_{k, g, m}$ for each codeword $(g, m)$. Then each user feeds back the maximum SINR value $\operatorname{SINR}_{k, g^{*}, m^{*}}$ and the corresponding codeword index $\left(g^{*}, m^{*}\right)$. The BS schedules users at $t^{\text {th }}$ time slot using the following procedures:

Step 1: For each codeword $(g, m)$, select the user $k^{*}$ making $S_{\text {INR }}$ maximum. Because the BS knows the CSI by limited feedback, the equal power allocation is suitable. So the maximum SINR means maximum the data rate of each user.

$$
k^{*}=\arg \max _{k} \operatorname{SINR}_{k, g, m}
$$

Step 2: For each sub-codebook $g$, compute sub-codebook capacity:

$$
R_{g}(t)=\sum_{m=1}^{N} R_{k^{*}, g, m}(t),
$$

where $R_{k^{*}, g, m}(t)=\log \left(1+P_{0} \operatorname{SINR}_{k^{*}, g, m}\right)$.

Step 3: Selecting $g^{*}=\arg \max R_{g}(t)$.

So the BS selects the sub-cođébook $g^{*}$ as pre-coding matrix, and the sum capacity of system is $R(t)=R_{{ }^{*}}(t)$

It can be seen that the user selection scheme is based on system sum capacity. If the PFS is considered, we can get another user selection scheme. So we modify the Step
1 and add the Step 4.

Step 1: For each codeword $(g, m)$, select the user $k^{*}$ making $\operatorname{SINR}_{k, g, m} / \mu_{k}(t)$ maximum.

$$
k^{*}=\arg \max _{k} \frac{S I N R_{k, g, m}}{\mu_{k}(t)},
$$

Step 4: Update the $\mu_{k}(t)$ using formula (8).

In the following, the first scheme is called Non-PFS scheme, and the second scheme is called PFS scheme.

The flow diagram of user selection with PFS can be showed in Figure 2. In the flow diagram, $i$ is sub-codebook index, $j$ is the codeword index in $i^{\text {th }}$ sub-codebook. $k$ is the user index. Here, we just show the process of one time slot.

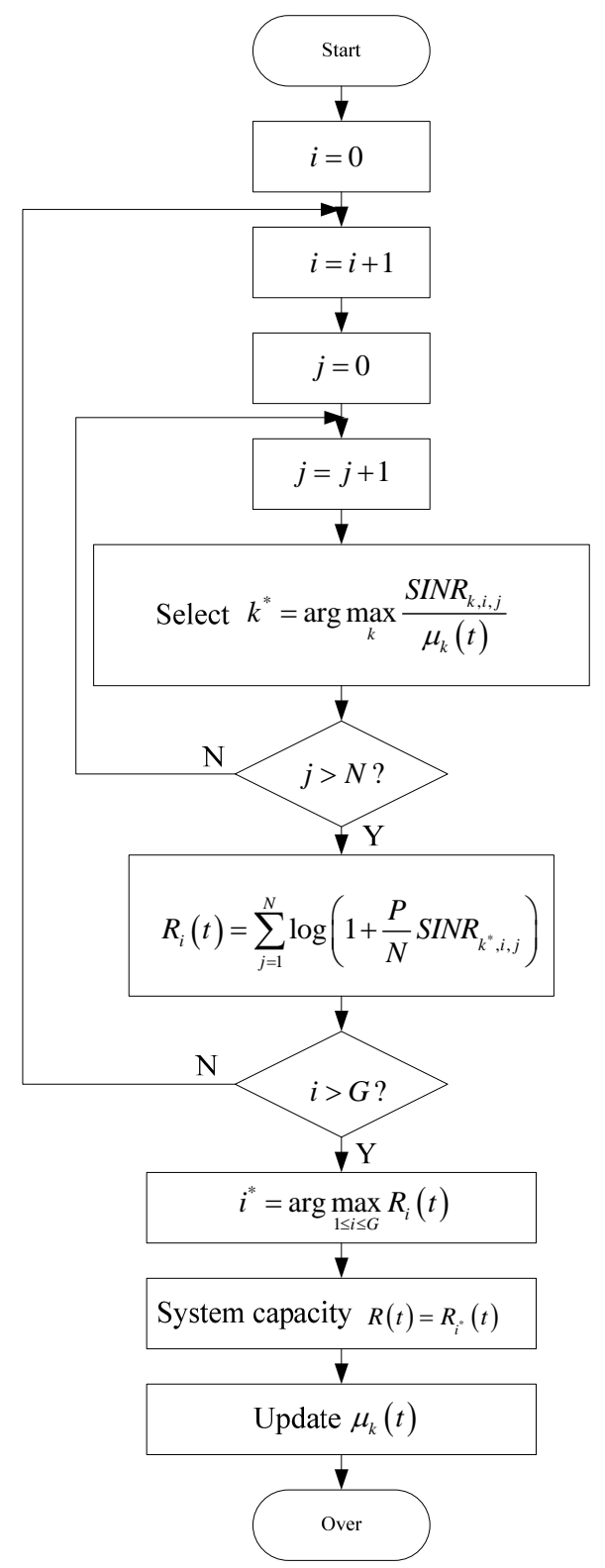

Figure 2. User selection flow diagram. 


\section{Simulation Result and Analysis}

Simulation results are showed in this section. Using LTE codebook, the PFS scheme is applied to observe the user fairness in the simulation. To compare users with different channel condition, we assume their channel coefficients from different complex Gaussian distribution, where a quarter of channels follow $C N(0,1)$, a quarter of $C N$ $(0,1 / 2)$, a quarter of $C N(0,1 / 4)$, and the other $C N(0,1 / 8)$. The BS is equipped with $N=4$ antennas, and each user has single antenna. The user is assumed to know its own CSI perfectly. The transmitting power constraint is $P=10$ $\mathrm{dB}$. The size of window is $t_{c}=100$. We use Monte- Carlo simulations to plot the figures with 100,000 times.

Figure 3 shows me the curve of system sum capacity with different users on different feedback bits without considering PFS. We can conclude that both increasing the number of user number and feedback bit number can improve system sum capacity, but along with the linear increasing of the two factors, the improvement of system sum capacity tends to flat. That is because the system capacity tends to saturation when user number and feedback bit number get certain value, then further increase the number of user and feedback bit can not improve system capacity.

Figure 4 shows the system sum capacity with 2 feedback bits and 4 feedback bits respectively. And the x-axis is the number of user. From the figure, one can observe that when the number of feedback bit is fixed, the Non-PFS scheme achieves more sum capacity than the PFS. This is because the PFS scheme takes the user fairness into account. Besides, we can also see that the difference between Non-PFS scheme and PFS scheme become larger along with the increace of user number. This is because when the number of user is big enough, the BS has more users to choose. The PFS scheme need to take care of the more bad channel users, but the Non-PFS scheme select the user just considering the capacity. So the differece is larger along with the increace of user number. Besides, the two PFS schemes have a crosspoint. It signifies that the system sum capacity of 2 bit feedback PFS scheme is higher than 4 bit when the user number is small, but at the same time, the user fairness will be lower.

Figure 5 and Figrue 6 show the fairness of 16 users. In this paper, we take the average data rate of each user as the criterion to measure the user fairness. We can clearly see that compared with Non-PFS scheme, the bad channel user of PFS scheme gets better service and achieve much more average data rate. Moreover, it is observed that the user fairness with 2 feedback bits is better than that with 4 feedback bits. This is due to the fact the number of user is not large enough. When the number feedback bit is 2, the number of LTE sub-codebook is 1 , and the number of LTE sub-codebbok is 4 for

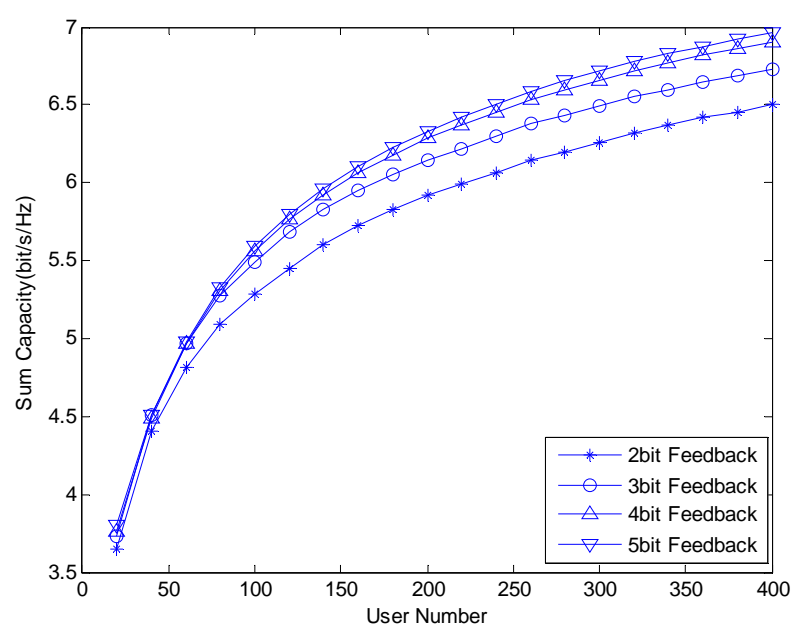

Figure 3. System sum capacity versus the number of users on different feedback bits.

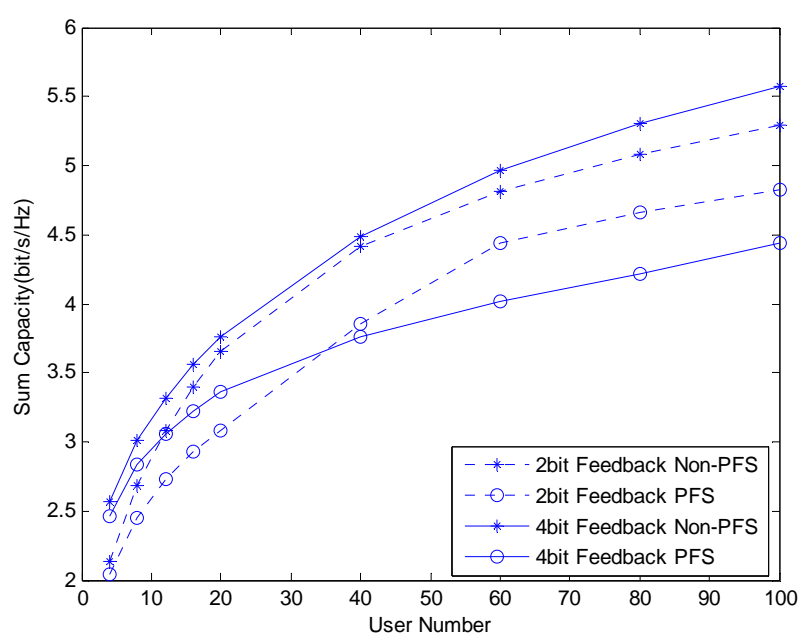

Figure 4. The system sum capacity in different user number.

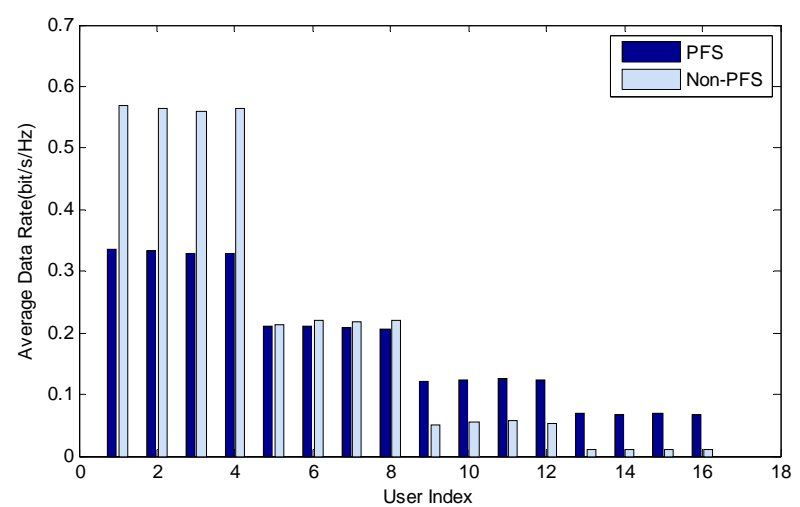

Figure 5. The average data rate of each user ( 2 feedback bits).

4 feedback bits. If the number of user is fixed, the virtual user number of each sub-codebook is much smaller for 4 feedback bits scheme, so the BS may not be select 


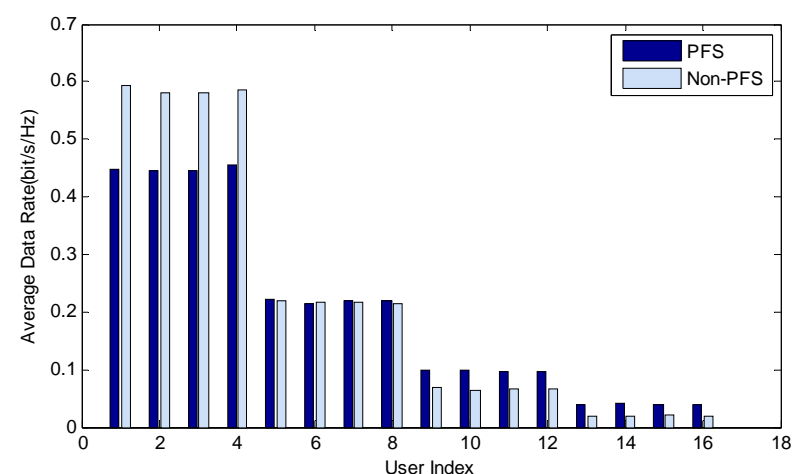

Figure 6. The average data rate of each user (4 feedback bits)

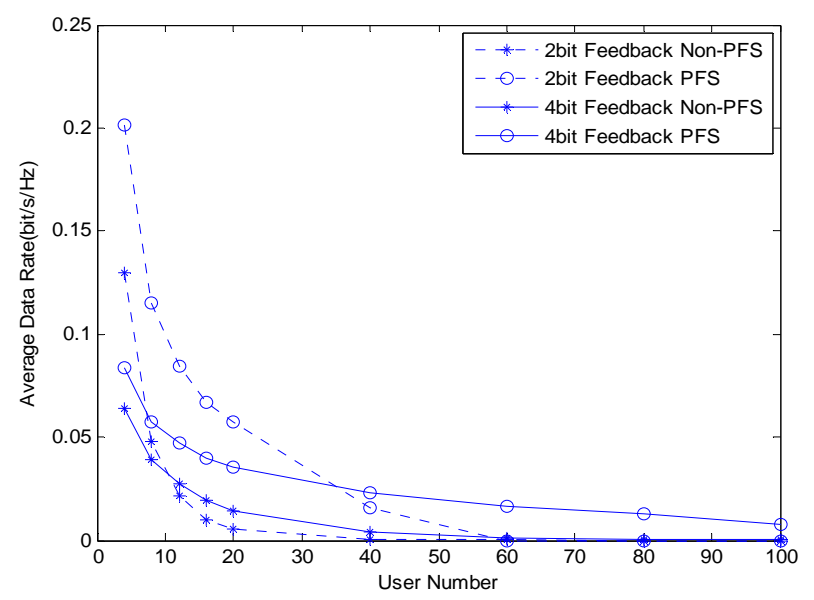

Figure 7. Bad channel user average data rate in different user number.

enough users, it is helpful to good channel user. So it can weaken the fairness. The simulation result of different user number is demonstrated in Figure 7.

Next, we will analyze how the varying number of user influences the user fairness. Here, we only consider the bad channel users whose channel coefficient is from $C N(0,1 / 8)$ distribution. The simulation result of average data rate of bad channel users with varying number of users is shown in Figure 7. From the figure, we can summary that, when the number of user is less than 35, the bad channel user data rate of 2 bits feedback PFS scheme is better than 4 bit feedback PFS scheme, when the number of user is larger than 35, the bad channel user data rate of 4 bits feedback PFS scheme is better than 2 bit feedback PFS scheme. It indicates that 2 bits feedback PFS scheme leads to the better fairness when the number of user is small and 4 bits feedback PFS scheme leads to the better fairness when the number of user is large. So in LTE system, when the number of user is small, we can achieve better user fairness by choosing less feedback bits; on the other hand, when the number of users is large, we can obtain higher fairness by choosing more feedback bits.

\section{Conclusions}

In this paper, we studied the performance of a generalized PFS scheme in limited feedback multi-user MIMO downlink system, with the LTE codebook as local codebook. It can be concluded that the PFS scheme can get the tradeoff between sum capacity and user fairness. We further analyzed the influence of the number of users and the number of feedback bits on user fairness and capacity. The simulation results show that, when the number of users is small, we can use fewer bits to feedback user's CSI to achieve better user fairness, while more bits should be used when the number of users is large.

\section{Acknowledgements}

This work is partly supported by the National Science and Technology Major Project (2012ZX03004003) and China National Science Foundation under Grand No.61201148.

\section{REFERENCES}

[1] 3GPP, "Evolved Universal Terrestrial Radio Access (E-UTRA); Physical channels and modulation,” 3GPP TS 36.211 version 10.0.0 Release 10 .

[2] G. Caire and S. Shamai, "On the Achievable Throughput of a MultiantennaGassian Broadcast Channel,” IEEE Transactions on Information Theory, Vol. 49, 2003, pp. 1691-1706. doi:10.1109/TIT.2003.813523

[3] Q. H. Spencer, A. L. Swindlehurst and M. Haardt, “Zero-Forcing Methods for Downlink Spacial Multiplexing in Multiuser MIMO Channels," IEEE Transactions on Signal Process, Vol. 52, No. 2, 2004, pp. 461-471. doi:10.1109/TSP.2003.821107

[4] Masoud and B. Hassibi, "On the Capacity of MIMO Broadcast Channels with Partial Side Information”, IEEE Transanctions on Information Theory, Vol. 51, 2003, pp. 506-522.

[5] T. Yoo, N. Jindal and A. Goldsmith, "Multi-antenna Downlink Channels with Feedback and User Selection," IEEE J. Select. Areas Commun., Vol. 25, No. 7, 2007, pp. 1478-1491. doi:10.1109/JSAC.2007.070920

[6] K. Huang, A. Texas Univ., R. W. Hearth and J. G. Andrews, "Space Division Multiple Access with a Sum Feedback Rate Constraint,” IEEE Transactions on Signal Process, Vol. 55, No. 7, 2007, pp. 3879-3891. doi:10.1109/TSP.2007.894245

[7] Y. X. Wang, S. Y. Hur, Y. W. Park and J.-H. Choi, "Efficient User Selection Algorithms for Multiuser MIMO Systems with Zero-forcing Dirty Paper Coding," IEEE Journal of Communications and Networks, Vol. 13, 2011, pp. 232-239.

[8] T. Yoo and A. Goldsmith, "On the Optimality of Multiantenna Broadcast Scheduling Using Zero-forcing Beamforming," Ieee journal on Selected Areas in Communications, Vol. 24, No. 3, 2006, pp. 528-541. doi:10.1109/JSAC.2005.862421 
[9] N. Sharma, "A Study of Opportunism for Multiple-anrenna System,” IEEE Transactions on Information Theory, Vol. 51, No. 5, 2006, pp. 1804-1814. doi:10.1109/TIT.2005.846410

[10] Choi and Jeongsik, "User Thoughput Estimation for the
PF Scheduling Algorithm under MIMO Channel Environments,” IEEE Wireless Communications Letters, Vol. 1, 2012, pp. 528-531.

doi:10.1109/WCL.2012.072012.120455 